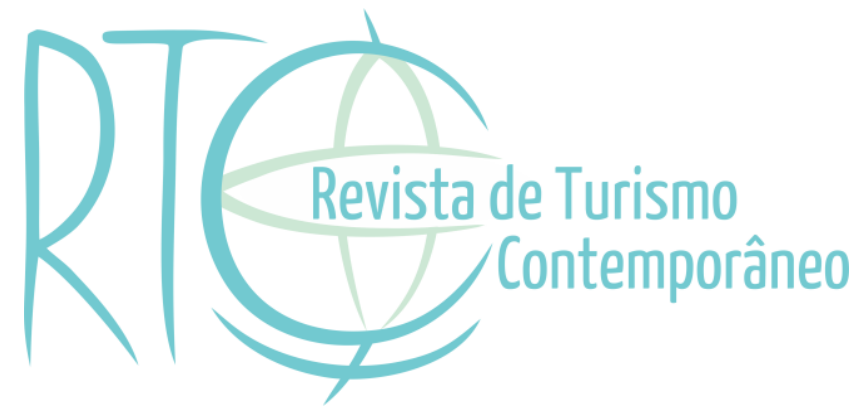

\title{
Consumo e Sustentabilidade em Turismo: panorama, conceitos e métodos aplicados no contexto de pesquisas no Brasil
}

Consumption and Sustainability in Tourism: panorama, concepts and methods applied in research developed in Brazil

Sonia Rebouças da Silva Melo

Professora adjunta no Centro Acadêmico do Agreste (CAA) da Universidade Federal de Pernambuco - UFPE, Caruaru/PE, Brasil

E-mail: soniareboucas@gmail.com

Minelle Enéas da Silva

Professor associado em Sustentabilidade da Cadeia de Suprimentos na Excelia Business School - Excelia, La Rochelle/França

E-mail: minele.adm@gmail.com

Francisco Vicente Sales Melo

Professor adjunto do Departamento de Administração da Universidade Federal do Ceará UFC, Fortaleza/CE, Brasil

E-mail: vicsmelo@gmail.com 


\section{RESUMO}

Os conceitos de consumo associados à sustentabilidade geralmente são múltiplos, porém imprecisos e com pouco consenso. No contexto de pesquisas em turismo, isso se torna ainda mais evidente, uma vez que esta área do conhecimento exige um pensamento multidisciplinar. Com foco no conceito de consumo sustentável, o objetivo central deste artigo é compreender como consumo e sustentabilidade são utilizados em pesquisas na área do turismo. Para tanto, foi realizada uma revisão sistemática da literatura de 71 publicações acadêmicas brasileiras no período de 2008 a 2017. Os resultados revelaram que o conceito de consumo utilizado na área de turismo em estudos sobre sustentabilidade tende a ser amplo e, comumente, a definição aborda mais aspectos ambientais e econômicos. Observa-se que nas pesquisas considerando a perspectiva prática da preservação, há uma tendência a não considerar os turistas como agentes desse processo de preservar, isso porque o consumo, nesse contexto, normalmente está voltado para análise das escolhas que as pessoas fazem ao decidirem por um bem ou serviço. Nesse sentido, a ideia de associar o ato de consumir às práticas de sustentabilidade nas destinações é uma das principais propostas para agenda de pesquisas da área.

Palavras-chave: Consumo Sustentável. Turismo. Sustentabilidade em Turismo. Publicações Nacionais.

\section{ABSTRACT}

The concepts linking consumption and sustainability are generally multiple, but imprecise and with little consensus. In the context of tourism research, this becomes even more evident, since this area of knowledge requires multidisciplinary thinking. Focusing on the concept of sustainable consumption, the main objective of this article is to understand how consumption and sustainability are used in the research field of tourism. To this end, a systematic literature review of 71 Brazilian academic publications was carried out from 2008 to 2017. The results revealed that the concept of consumption used in the field of tourism in studies on sustainability tends to be broad and, commonly, the definition addresses more environmental and economic aspects. It is observed that in research considering the practical perspective of preservation, there is a tendency to not consider tourists as agents of this preservation process, because consumption, in this context, is usually aimed at analyzing the choices that people make when deciding for a good or service. In this sense, the idea of associating the act of consuming with sustainability practices in destinations is one of the main proposals for the research agenda in the area.

Keywords: Sustainable Consumption. Tourism. Sustainable Tourism. Brazilian Publications. 


\section{INTRODUÇÃO}

O conceito de consumo, assim como o de sustentabilidade, vem sendo utilizado de diversas formas por pesquisadores e interessados no assunto. Apesar de existirem definições que tendem a abrangê-los de maneira mais completa ao considerar as dimensões econômicas, sociais, ambientais e culturais, como os de Hunter (2002) e Ciegis, Ciegis e Jasinskas (2005), ainda se têm diversas perspectivas sendo utilizadas, o que muitas vezes pode dificultar a compreensão e aplicação prática de tais conceitos por parte dos interessados na temática. No âmbito das investigações em turismo, essas variações de definição também não são exceção, o que gera a necessidade de se buscar um alinhamento conceitual e prático, dadas as especificidades da área.

Obter um panorama de como está parte da produção científica brasileira sobre sustentabilidade na área do turismo, bem como observar como a perspectiva do consumidor é inserida nas análises e quais conceitos são normalmente utilizados, pode contribuir com o rumos das pesquisas nesse campo, já que a falta de entendimento conceitual pode levar a dificuldades relacionadas à implantação prática de ações voltadas à sustentabilidade. Pesquisas já verificaram como o tema sustentabilidade vem sendo trabalhado em publicações brasileiras (Junqueira, Pinheiro \& Maior, 2012; Machado Jr. et al., 2013; Silva, Alves \& Volkmer, 2014; Luca, Cardoso, Vasconcelos \& Pontes, 2014). No entanto, no contexto do turismo, não se observou trabalhos com essa finalidade, sobretudo estudos com ênfase na identificação dos conceitos de consumo associados à perspectiva da sustentabilidade.

Se por um lado a sustentabilidade de/em uma destinação turística é uma preocupação da área, por outro, deve-se haver a preocupação de como os estudos vêm sendo desenvolvidos de modo a contribuir com o entendimento dessa sustentabilidade, o que pode se dá a partir de análises voltadas para o desenvolvimento de métodos e técnicas de preservação e manutenção. Levando-se em conta os seus três principais pilares da sustentabilidade (econômico, social e ambiental), Luca et al. (2014) consideraram que o debate acerca dos temas relacionados à sustentabilidade e o interesse por parte das empresas, governos e consumidores encontram-se em franco desenvolvimento, tanto no meio acadêmico quanto no contexto empresarial; afirmativa que ainda pode ser considerada atual.

Neste contexto, o presente estudo tem por objetivo compreender como consumo e sustentabilidade são utilizados em pesquisas na área do turismo; isso porque o consumo associado à prática da sustentabilidade por parte dos turistas é algo de extrema relevância para os estudos em turismo. Além disso, há vasta literatura analisando a sustentabilidade de 
destinações turísticas, no entanto, parte-se do pressuposto de que abordagens que tratam de forma ampla o comportamento 'sustentável' do consumidor enquanto visitantes e o consumo em si, ainda são incipientes, não sendo apenas uma lacuna voltada ao desentendimento conceitual, mas também, de aplicação.

Acredita-se que o alinhamento do entendimento do que significa sustentabilidade a partir da literatura do turismo seja uma primeira etapa relevante para implantação de projetos práticos para preservação de localidades turísticas. Desse modo, a presente pesquisa contribui para a literatura no sentido de buscar clareza na aplicação dos conceitos, o que pode refletir na ação dos atores envolvidos com o tema. A pesquisa é apresentada em quatro seções além desta introdutória. A seguir tem-se uma breve revisão sobre consumo e sustentabilidade, seguido pelos procedimentos metodológicos adotados na pesquisa. Os resultados são apresentados de acordo com as principais categorias: periódicos e qualificação; ênfase, enfoque e tipologias de consumo em relação à sustentabilidade; métodos usados e objetivos, e finalidades. O estudo é finalizado com as considerações finais.

\section{CONSUMO E SUSTENTABILIDADE NO TURISMO}

Com o crescimento da produção mundial e, consequentemente, do consumo, especialistas, governantes e organizações de vários setores, sobretudo aqueles orientados às questões ambientais, começaram a considerar o impacto das atividades de consumo de indivíduos sobre o meio ambiente (Eden, 1993). Pesquisadores passaram a considerar que ações individuais conscientes, bem informadas e preocupadas com as questões ambientais, poderiam ser uma nova estratégia para a solução dos problemas ligados ao meio ambiente e para as mudanças em direção à sociedade sustentável (Eden, 1993; Halkier, 1999; Portilho, 2005). Atividades simples e cotidianas como fazer compras de produtos considerados básicos, assim como de itens considerados luxuosos, começaram a serem vistas como comportamentos e escolhas que podem afetar a qualidade do meio ambiente, surgindo, assim, a necessidade de um consumo mais verde (Portilho, 2005). Ou seja, não apenas o consumo verde, mas também o consciente (individual), o responsável ou o sustentável (coletivo) seriam boas formas de contribuir com preservação do meio ambiente (Silva, Oliveira \& Gómez, 2013).

No entanto, entende-se que apenas a redução e substituição do consumo de produtos não seria suficiente para uma sociedade mais sustentável. É razoável que ações individuais estejam sempre alinhadas à ações coletivas (Silva, 2012), assim como na implementação de políticas multilaterais de regulação, tanto da produção quanto do consumo (Beckerman, 1992; Portilho, 2005). Portilho (2005, p. 4) destaca que a tática de se estabelecer produção e 
consumo verdes começa a perder ênfase em nome de uma estratégia de produção e consumo sustentáveis. Para a autora, "o meio ambiente deixou de ser relacionado apenas a uma questão de como usamos os recursos (os padrões), para também estar vinculado à preocupação com o quanto usamos (os níveis); portanto, um problema de acesso, distribuição e justiça”.

No contexto do turismo, entende-se que o consumo é ainda mais complexo, pois essa perspectiva vai do consumo de bens e serviços na destinação até a exploração dos atrativos turísticos, na maioria das vezes, naturais. Estudos vêm indicando que os indivíduos são positivamente influenciados por comportamentos de consumo mais ambientalmente corretos (Manaktola \& Jauhari, 2007; Melo \& Farias, 2014). No entanto, o comportamento de consumo verde ou consciente, de modo a englobar no mínimo as dimensões econômicas, sociais e ambientais, ainda é pouco comum entre os consumidores, sobretudo os turistas. Isso ocorre porque normalmente os turistas estão muito mais preocupados em explorar e conhecer uma destinação do que preservá-la (Melo \& Farias, 2018).

A discussão sobre consumo sustentável no turismo é complexa por se tratar de algo que pode ser considerado como uma opção para preservação do local. Porém, ao mesmo tempo, tende a ser criticado por ser algo limitante ao crescimento e desenvolvimento. Ou seja, só o fato de uma área ser explorada já pode torná-la insustentável. A princípio é preciso se ter definições claras e propostas concretas para que os responsáveis pelas destinações possam criar estratégias para melhorar o processo de manutenção e preservação local, sobretudo para aqueles em que os atrativos são naturais. Para tanto, é preciso compreender a forma como os estudos na área de turismo focam consumo e sustentabilidade.

\section{PROCEDIMENTOS METODOLÓGICOS}

Para atender ao objetivo proposto, adotou-se o método sistemático de revisão bibliográfica, sendo o estudo do tipo descritivo de abordagem quali-quantitativa (Gil, 2008). A revisão da literatura permite, ao pesquisador, apontar e avaliar o conhecimento produzido em pesquisas anteriores. Neste sentido, Castro (2010, p. 1) afirma que a revisão sistemática "é uma revisão planejada para responder a uma pergunta específica e que utiliza métodos explícitos e sistemáticos para identificar, selecionar e avaliar criticamente os estudos, e para coletar e analisar os dados destes estudos incluídos na revisão".

Seguindo as três etapas do método sistemático descrito por Clarke e Oxman (2001), iniciou-se a pesquisa com o planejamento da revisão a ser realizada. Após a revisão, estruturou-se o artigo para comunicação e divulgação. Para esta pesquisa, selecionou-se um período de 10 anos, os anos de 2008 a 2017, considerando o avanço nas pesquisas sobre 
sustentabilidade. A pesquisa foi realizada entre outubro e dezembro de 2018 , utilizando-se da base de dados online SPELL (Scientific Periodicals Eletronic Library), voltada às áreas de Administração, Contabilidade e Turismo. As palavras-chave empregadas para a pesquisa foram: sustent* e consumo, sustent* e turismo, consumo, e turismo.

Em uma pesquisa inicial, um total de 195 artigos foram identificados. Iniciando a análise da base selecionada, identificou-se 24 artigos duplicados, restando 171 artigos. Neste primeiro momento o foco central foi identificar pesquisas sobre consumo e sustentabilidade. No entanto, quando o filtro para área de turismo foi inserido na base analisada, esse número reduziu para 104 trabalhos. Por fim, analisou-se aqueles artigos focados em turismo e sustentabilidade que de alguma forma discutem consumo, sendo essa quantidade reduzida para 71 artigos; quantidade final que constituiu a base de dados desta pesquisa. Vale ressaltar que, nessa seleção, não houve especificação em termos de qualificação da CAPES do periódicos nos quais os artigos foram publicados.

Após a seleção, os artigos selecionados foram catalogados, com o uso do software Excel, extraindo-se os dados que respondem à questão da pesquisa. Para melhor apresentar os resultados, uma lista foi criada a partir de codificações associadas às categorias de análise considerando a evolução temporal e os objetivos propostos (ver Apêndice). Nesta pesquisa, os dados foram analisados conforme as seguintes categorias: (1) quantidade de publicações; (2) periódicos e Qualis Capes; (3) ênfase, enfoque e tipologias de consumo em relação à sustentabilidade; (4) características metodológicas; e (5) objetivos e finalidades. Para tanto, foi realizada a análise do conteúdo dos artigos seguindo os critérios de Bardin (2009). Para garantir a confiabilidade dos resultados, a análise foi realizada de forma interativa entre os pesquisadores, no sentido de garantir o entendimento sobre o tema. Vale salientar que para facilitar a compreensão do contexto analisado, um nuvem de palavras foi desenvolvida para representar objetivos e finalidades dos artigos selecionados (Vilela, Ribeiro \& Batista, 2020).

\section{ANÁLISES}

Antes de iniciar a análise do conteúdo dos artigos selecionados, primeiramente foi feita uma análise em relação aos periódicos publicados e Qualis Capes (2013-2016). Com base nos 71 artigos que compõem a base de dados, constou-se que 54\% dos trabalhos foram publicados em periódicos com Qualis B1 e 21\% naqueles com Qualis B2. Em frequência menor, 15\% dos artigos acessados foram publicados em revistas Qualis A2 e 10\% em B3. Cabe destacar que no triênio de 2010 a 2012 apenas a Revista de Administração Pública era qualificada como A2 e o Caderno Virtual de Turismo como B1. Com a última revisão do 
Qualis (2013-2016), várias revistas da área de Administração Pública e de Empresas, Ciências Contábeis e Turismo foram mais bem qualificadas, o que de certa forma contribuiu para a valorização da publicação de artigos em periódicos brasileiros.

Tabela 1 - Artigos Publicados por Revista e Qualis Capes por Ano (2008-2017)

\begin{tabular}{|c|c|c|c|c|c|c|c|c|c|c|c|c|}
\hline \multirow[t]{2}{*}{ Revista } & \multirow{2}{*}{$\begin{array}{l}\text { Qualis } \\
\text { Capes }\end{array}$} & \multicolumn{10}{|c|}{ Ano } & \multirow{2}{*}{$\begin{array}{r}\text { Total por } \\
\text { Revista }\end{array}$} \\
\hline & & 2008 & 2009 & 2010 & 2011 & 2012 & 2013 & 2014 & 2015 & 2016 & 2017 & \\
\hline $\begin{array}{l}\text { Revista Brasileira de } \\
\text { Pesquisa em Turismo }\end{array}$ & A2 & 1 & 1 & 0 & 1 & 2 & 0 & 1 & 2 & 1 & 0 & 9 \\
\hline Revista de Adm. Pública & A2 & 0 & 0 & 1 & 0 & 1 & 0 & 0 & 0 & 0 & 0 & 2 \\
\hline $\begin{array}{l}\text { Administração Pública e } \\
\text { Gestão Social }\end{array}$ & B1 & 0 & 0 & 1 & 0 & 0 & 0 & 0 & 0 & 0 & 0 & 1 \\
\hline $\begin{array}{lll}\text { Caderno } & \text { Virtual } & \text { de } \\
\text { Turismo } & & \end{array}$ & B1 & 4 & 4 & 0 & 1 & 3 & 1 & 0 & 0 & 0 & 2 & 15 \\
\hline $\begin{array}{l}\text { Revista de Gestão Social } \\
\text { e Ambiental }\end{array}$ & B1 & 0 & 0 & 1 & 0 & 0 & 0 & 1 & 0 & 0 & 0 & 2 \\
\hline Turismo em Análise & $\mathrm{B} 1$ & 1 & 3 & 0 & 3 & 1 & 1 & 1 & 0 & 0 & 1 & 11 \\
\hline Turismo: Visão e Ação & B1 & 1 & 0 & 1 & 1 & 1 & 3 & 1 & 1 & 0 & 0 & 9 \\
\hline $\begin{array}{l}\text { Desenvolvimento em } \\
\text { Questão }\end{array}$ & B2 & 0 & 0 & 0 & 0 & 0 & 0 & 0 & 0 & 1 & 0 & 1 \\
\hline $\begin{array}{lr}\text { Reunir: Revista } & \text { Adm., } \\
\text { Contabilidade } & \text { e } \\
\text { Sustentabilidade } & \end{array}$ & B2 & 0 & 0 & 0 & 1 & 0 & 0 & 0 & 0 & 0 & 0 & 1 \\
\hline $\begin{array}{ll}\text { Revista Acadêmica } & \text { do } \\
\text { Observatório } & \text { de } \\
\text { Inovação do Turismo } & \end{array}$ & B2 & 2 & 0 & 1 & 1 & 0 & 0 & 0 & 0 & 1 & 1 & 6 \\
\hline Revista de & & & & & & & & & & & & \\
\hline $\begin{array}{l}\text { Administração } \\
\text { Unimep }\end{array}$ & B2 & 0 & 0 & 0 & 0 & 0 & 0 & 1 & 0 & 0 & 0 & 1 \\
\hline $\begin{array}{l}\text { Revista de Gestão e } \\
\text { Secretariado }\end{array}$ & $\mathrm{B} 2$ & 0 & 0 & 0 & 0 & 0 & 0 & 0 & 0 & 1 & 0 & 1 \\
\hline $\begin{array}{l}\text { Rosa dos Ventos - } \\
\text { Turismo e Hospitalidade } \\
\text { Interface - Revista do }\end{array}$ & $\mathrm{B} 2$ & 0 & 0 & 0 & 2 & 1 & 1 & 1 & 0 & 0 & 0 & 5 \\
\hline $\begin{array}{l}\text { Centro de Ciências } \\
\text { Sociais Aplicadas }\end{array}$ & B3 & 0 & 1 & 0 & 0 & 0 & 0 & 0 & 0 & 0 & 0 & 1 \\
\hline $\begin{array}{l}\text { Pensamento } \quad \& \\
\text { Realidade }\end{array}$ & B3 & 0 & 0 & 0 & 0 & 1 & 0 & 0 & 0 & 0 & 0 & 1 \\
\hline $\begin{array}{l}\text { Revista Brasileira de } \\
\text { Estratégia }\end{array}$ & B3 & 0 & 1 & 0 & 0 & 0 & 0 & 0 & 0 & 0 & 0 & 1 \\
\hline Revista Hospitalidade & B3 & 0 & 0 & 1 & 1 & 0 & 1 & 0 & 0 & 1 & 0 & 4 \\
\hline $\begin{array}{l}\text { Total de artigos } \\
\text { publicados por ano }\end{array}$ & & 9 & 10 & 6 & 11 & 10 & 7 & 6 & 3 & 5 & 4 & 71 \\
\hline
\end{tabular}

Fonte: Dados da Pesquisa, 2019.

Considerando as publicações a partir dos anos analisados, observou-se que $65 \%$ dos artigos sobre consumo e sustentabilidade, foram publicados de 2008 a 2012, tendo-se um menor percentual de publicações (35\%) entre os anos de 2013 e 2017 (Tabela 1). Tal fato demonstra ter havido menor interesse nos últimos anos sobre a temática, algo que poderia ser verificado como uma lacuna ou uma oportunidade para o desenvolvimento de pesquisa, uma vez que o tema tem se tornado cada vez mais recorrente em outras áreas. Outro dado 
interessante é que 15,5\% dos artigos foram publicados em periódicos com Qualis A2 e 53,5\% B1, o que indica um percentual significativo de artigos em revistas bem qualificadas. Em seguida, apresentam-se aspectos relacionados a ênfase e enfoque da temática em questão.

\section{1. Ênfase, enfoque e tipologias de consumo em relação à sustentabilidade}

Inicialmente, como apresentado na Tabela 2, observou-se que boa parte dos estudos $(39,4 \%)$ não dão ênfase às características e dimensões comuns da sustentabilidade. Ou seja, não especificam as dimensões utilizadas, o que dificulta o entendimento do contexto e lógica analisada. A ênfase à perspectiva sustentável, que é aquela relacionada as dimensões que a compõe (de forma geral), aparece em 22,5\% dos estudos. A dimensão econômica (16,9\%) e a dimensão ambiental (11,3\%) também aparecem de maneira conjunta $(8,5 \%)$ nos artigos. Ressalta-se que apenas um dos artigos analisados (artigo 35) considerou como aspecto central a dimensão social da sustentabilidade no contexto turístico investigado. Além disso, constatou-se que $63,4 \%$ dos artigos enfatizam a sustentabilidade como enfoque principal, sem especificações de dimensões, enquanto $33,8 \%$ tratam o tema em uma perspectiva desenvolvimentista direcionada aos princípios econômicos.

Tabela 2 - Ênfase Atribuída às Características de Sustentabilidade nos Estudos em Turismo

\begin{tabular}{llll}
\hline \multicolumn{1}{c}{ Ênfase Atribuída } & N & \multicolumn{1}{c}{$\%$} & \multicolumn{1}{c}{ Códigos dos Artigos } \\
\hline Sem especificação & 28 & 39,4 & $1,2,7,11,13,14,15,16,19,31,32,34,47,48,51,53,54,58$, \\
& 16 & 22,5 & $4,5,6,62,63,65,67,68,69,70,71$ \\
Sustentável & 12 & 16,9 & $10,21,25,28,38,42,43,45,49,50,56,60,64$ \\
Econômica & 8 & 11,3 & $3,8,20,36,37,40,42,66$ \\
Ambiental & 6 & 8,5 & $9,27,30,41,44,46$ \\
Ambiental e econômica & 1 & 1,4 & 35 \\
Social & $\mathbf{7 1}$ & $\mathbf{1 0 0}$ & \\
Total & & $\mathbf{T}$ & \\
\hline
\end{tabular}

Fonte: Dados da Pesquisa, 2019.

No que se refere às tipologias de consumo utilizadas, cabe ressaltar que o consumo em si não é foco central nas pesquisas sobre turismo e sustentabilidade. Nesse sentido, verificouse que $62 \%$ delas (Tabela 3) citam a ideia de consumo sustentável, enquanto apenas uma menciona consumo consciente e outra consumo de maneira geral. O que chamou atenção foi que $35,2 \%$ não apresentaram nenhuma especificação quanto à tipologia de consumo, o que deixa os estudos amplos e sem objetividade. A questão é que apesar de se abordarem a perspectiva do consumo consciente e sustentável, não se tem definições operacionais (aquelas que determinam a forma de mensuração, como escalas ou esquemas tipológicos) que possam determinar o que seria a consciência e a sustentabilidade no consumo. Espera-se que os 
estudos possam deixar mais claros esses conceitos e definições de modo a direcionar melhor o entendimento desses construtos na área.

Tabela 3 - Tipologias de Consumo Identificadas no Estudos em Turismo

\begin{tabular}{|c|c|c|c|}
\hline $\begin{array}{l}\text { Tipologia de Consumo } \\
\text { Utilizadas em Turismo }\end{array}$ & $\mathbf{N}$ & $\%$ & Códigos dos Artigos \\
\hline Consumo sustentável & 44 & 62,0 & $\begin{array}{l}1,3,4,5,6,8,9,10,12,17,18,20,21,22,23,24,25,26,27,28, \\
29,30,33,35,36,37,38,39,40,41,42,43,44,45,46,49,50,52, \\
55,56,57,60,64,66\end{array}$ \\
\hline Consumo consciente & 1 & 1,4 & 2 \\
\hline Consumo geral & 1 & 1,4 & 15 \\
\hline Sem especificação & 25 & 35,2 & $\begin{array}{l}7,11,13,14,16,19,31,32,34,47,48,51,53,54,58,59,61,62, \\
63,65,67,68,69,70,71\end{array}$ \\
\hline Total & 71 & 100 & \\
\hline
\end{tabular}

Fonte: Dados da Pesquisa, 2019.

Por outro lado, já se observa que a área de turismo parece estar alinhada à tipologia mais ampla de consumo relativa à sustentabilidade (ver Tabela 3). Além disso, é comum ver o conceito de consumo sustentável sendo associado a uma ou duas dimensões de sustentabilidade e, o consumo consciente ou verde, relacionado a preservação ambiental. Desse modo, pôde-se constatar que mesmo estando alinhado aos contextos atuais de consumo e sustentabilidade, ainda há confusão no uso desses conceitos na área do turismo.

Após a leitura dos textos, observou-se ainda que a perspectiva do consumo tende a não considerar o visitante como agente do processo de preservação de uma localidade. Ou seja, o visitante normalmente é visto como cliente que irá consumir os atrativos, não sendo responsável direto pela preservação dos espaços explorados. Por exemplo, a demarcação de área, limites de acessos e taxas de permanência, nem sempre envolvem o turista na prática da sustentabilidade, sendo essas ações apenas utilitárias. A proposta seria ir além dessas ações a partir da inclusão de outras que possam gerar mais envolvimento por parte do consumidorturista.

\subsection{Características Metodológicas das pesquisas analisadas}

No que se refere às abordagens de pesquisas utilizadas, verificou-se que $77,5 \%$ dos estudos sobre consumo e sustentabilidade são realizadas por meio de abordagens qualitativas e apenas 7,0\% quantitativas. Do total, 15,5\% são com abordagem quali-quantitativa ou mista, sendo que a prevalência da análise é qualitativa. Esse dado é interessante, pois indica um viés metodológico da área, podendo ser esse um tema interessante de investigação. Apesar de existirem várias possibilidades de pesquisas quantitativas na área de turismo, é possível supor que a abordagem qualitativa é predominante. 
Adicionalmente, constatou-se que $52,1 \%$ dos estudos foram caracterizados como do tipo exploratório, 19,7\% descritivos e 4,2\% explicativos (ver Tabela 4). Vale ressaltar que do total de artigos analisados, 23,9\% não especificaram o tipo de pesquisa adotado no método. Apesar de fazer esse levantamento, uma análise qualitativa dos artigos permite considerar que nem sempre os estudos considerados como exploratórios são de fato exploratórios. Essa classificação tem sido comum em trabalhos nas ciências sociais aplicadas, sendo uma prática inadequada em termos de classificação metodológica. Existe certa confusão quanto à aplicabilidade de conceitos metodológicos, o que sugere para novos pesquisadores na área a necessidade de delinear mais claramente suas propostas de pesquisa.

Tabela 4 - Tipos de Pesquisas Utilizadas

\begin{tabular}{llll}
\hline \multicolumn{1}{c}{ Tipo de Pesquisa } & \multicolumn{1}{c}{$\mathbf{N}$} & \multicolumn{1}{c}{$\%$} & \multicolumn{1}{c}{ Códigos dos Artigos } \\
\hline Exploratório & 37 & 52,1 & $14,16,17,18,19,20,21,22,23,24,25,27,28,31,36,40,41,42,44$, \\
& 14 & 19,7 & $3,4,48,49,51,52,53,54,56,58,59,60,62,64,65,67,68,69,71$ \\
Descritivo & 3 & 4,2 & $11,30,45$ \\
Explicativo & 17 & 23,9 & $1,2,5,6,7,8,9,10,12,13,15,26,33,34,38,43,66$ \\
Sem especificação & $\mathbf{7 1}$ & $\mathbf{1 0 0}$ & \\
& &
\end{tabular}

Fonte: Dados da Pesquisa, 2019.

Já no que se refere aos métodos utilizados, 33,8\% dos estudos são bibliográficos ou documentais, $18,3 \%$ estudos de casos e 9,9\% pesquisas de campo. Alinhado à abordagem quantitativa pouco utilizada, somente $5,6 \%$ são do tipo survey. Salienta-se ainda que apenas um estudo (artigo 53) foi elaborado por meio de pesquisa-ação. Constatou-se ainda que 31,0\% das investigações não definem método (ver Tabela 5). Retoma-se a necessidade de debater sobre mais rigor metodológico em pesquisas que discutem, na área de turismo, sobre consumo e sustentabilidade.

Tabela 5 - Métodos Utilizados

\begin{tabular}{|c|c|c|c|}
\hline Métodos & $\mathbf{N}$ & $\%$ & Códigos dos Artigos \\
\hline Bibliográfico / documental & 24 & 33,8 & $\begin{array}{l}2,3,8,11,12,13,16,19,21,29,32,33,36,38,39,47,49,50, \\
54,57,62,64,66,71\end{array}$ \\
\hline Survey & 4 & 5,6 & $1,9,55,70$ \\
\hline Estudo de caso & 13 & 18,3 & $4,5,7,10,18,27,30,31,34,37,48,61,63$ \\
\hline Pesquisa de campo & 7 & 9,9 & $6,17,28,43,45,59,60$ \\
\hline Pesquisa-ação & 1 & 1,4 & 53 \\
\hline Não define método & 22 & 31,0 & $\begin{array}{l}14,15,20,22,23,24,25,26,35,40,41,42,44,46,51,52,56, \\
58,65,67,68,69\end{array}$ \\
\hline Total & 71 & 100 & \\
\hline
\end{tabular}

Fonte: Dados da Pesquisa, 2019. 
A questão da não especificação dos tipos de pesquisa e dos métodos utilizados podem dificultar a continuidade de investigações de acordo com os procedimentos que vêm sendo empregados nas análises, visto que o progresso científico tende a ser tanto por meio de réplicas de pesquisas a partir da continuidade de análises quanto a partir de teorias e métodos que são usados. É necessário, assim, observar a epistemologia do método selecionado. Com base nos artigos analisados, parece que essa classificação não foi uma preocupação dos pesquisadores, algo que pode ser considerado como prejudicial ao desenvolvimento científico da área. Emerge, assim, como aspecto central para o desenvolvimento de novas pesquisas na área de turismo um melhor detalhamento e clareza metodológica, o que demonstra preocupação em minimizar as confusões teóricas já existentes.

Como análise complementar, observou-se que parte expressiva dos estudos trabalham o turismo de forma geral $(50,7 \%)$, sem especificar a atividade. O ecoturismo $(14,1 \%)$, o turismo rural $(7,0 \%)$, o comunitário $(5,6 \%)$ e o cultural $(4,2 \%)$ foram tipos um pouco mais utilizados nas análises. Como observado na Tabela 6, várias atividades turísticas aparecem, mas, com menos frequência, sugerindo que os pesquisadores da área tendem a trabalhar o turismo de forma abrangente.

Tabela 6 - Tipos de Atividade Turística

\begin{tabular}{llll}
\hline \multicolumn{1}{c}{ Tipo de Turismo } & $\mathbf{N}$ & $\mathbf{\%}$ & \multicolumn{1}{c}{ Códigos dos Artigos } \\
\hline Turismo em geral & 36 & 50,7 & $2,5,8,9,10,11,13,15,17,19,20,21,22,24,29,32,33,36,37,39$, \\
& & & $40,43,45,46,47,49,50,52,53,57,65,66,68,69,70,71$ \\
Ecoturismo & 10 & 14,1 & $1,3,23,26,31,54,59,60,61,64$ \\
Turismo Rural & 5 & 7,0 & $4,27,41,55,56$ \\
Turismo Cultural & 3 & 4,2 & $16,51,63$ \\
Turismo Sol e Praia & 2 & 2,8 & 6,25 \\
Turismo Indígena & 2 & 2,8 & 7,34 \\
Turismo Sexual & 1 & 1,4 & 67 \\
Turismo Comunitário & 4 & 5,6 & $12,28,30,35$ \\
Turismo Quilombola & 1 & 1,4 & 48 \\
Turismo de Eventos & 1 & 1,4 & 18 \\
Turismo Urbano & 1 & 1,4 & 58 \\
Turismo Gastronômico & 1 & 1,4 & 14 \\
Enoturismo & 1 & 1,4 & 31 \\
Turismo Social & 1 & 1,4 & 42 \\
Turismo Alternativo & 2 & 2,8 & 44,62 \\
Total & $\mathbf{7 1}$ & $\mathbf{1 0 0 , 0}$ & \\
\hline
\end{tabular}

Fonte: Dados da Pesquisa, 2019. 


\subsection{Objetivos e finalidades}

Considerando os objetivos e as finalidades das pesquisas analisadas sobre turismo e sustentabilidade no âmbito do consumo, uma nuvem de palavras foi criada para facilitar a compreensão do campo investigado (Figura 1). As principais palavras e termos que compõem os objetivos propostos nesses estudos são: turismo sustentável, desenvolvimento sustentável, impacto do turismo, práticas de sustentabilidade e práticas de gestão. Só dois artigos analisaram o comportamento dos consumidores enquanto turistas, algo que chama atenção já que se defende os turistas como agentes relevantes para o processo de preservação das localidades. Observa-se ainda que consumo ecologicamente consciente, análise de políticas públicas, análise do turismo de base comunitária e educação para sustentabilidade também são trabalhados, só que bem menos frequentes.

Figura 1 - Nuvem de palavras a partir dos objetivos das pesquisas

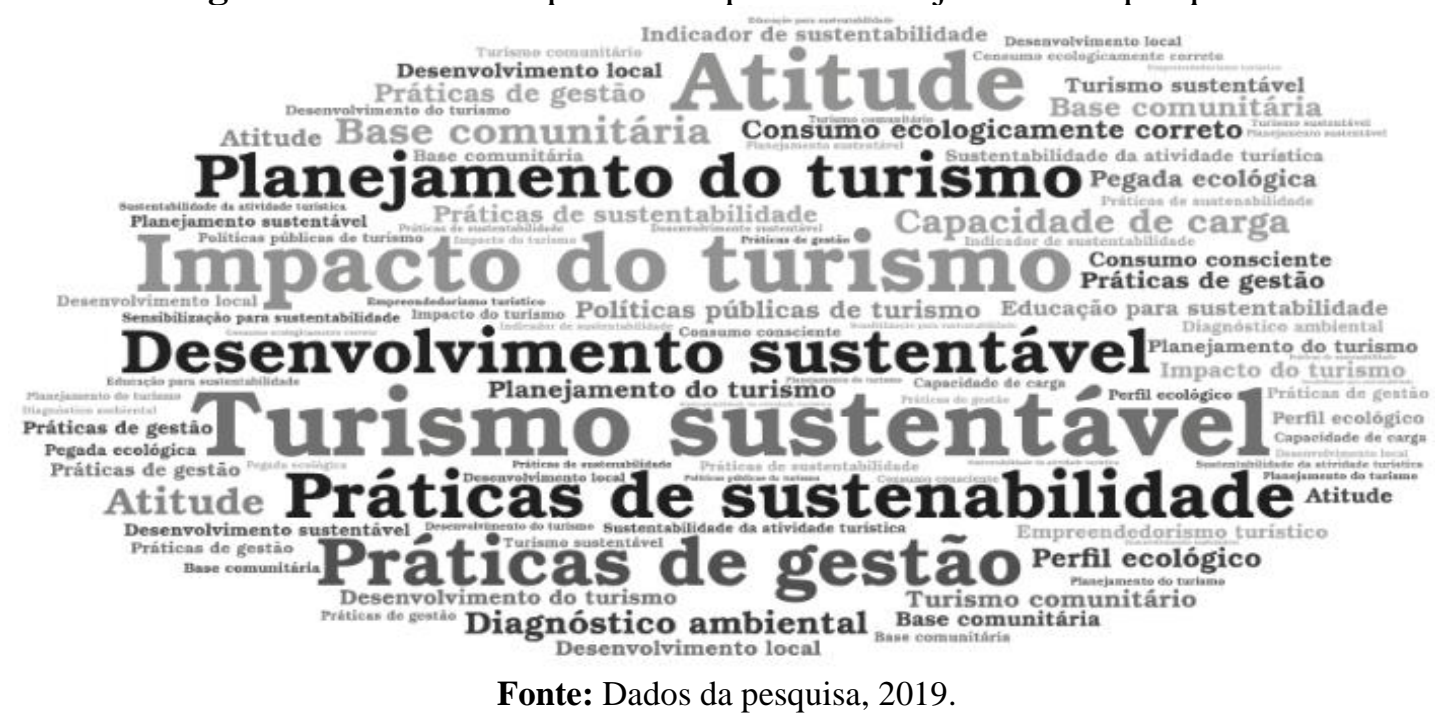

Quanto a finalidade, observou-se que poucas investigações mensuram o impacto da atividade turística nas localidades e avaliam o desenvolvimento local por meio de turismo de base comunitária. Em se tratando de métodos para se alcançar a sustentabilidade, as práticas de sustentabilidade, os diagnósticos de gestão da atividade turística e a mensuração e análise da capacidade de carga de destinações turísticas, também são objetivos que aparecem em determinados estudos, mas também não são frequentes (ver Figura 1).

Observou-se também que o consumo praticamente não aparece nos objetivos a partir da nuvem de palavras, indicando realmente que esse não tem sido foco nas pesquisas sobre sustentabilidade e turismo. Acredita-se que a perspectiva do consumo tende a ser ampla e relevante para compreensão das práticas de sustentabilidade nas destinações. Destarte, olhares 
com esse ponto de vista podem ampliar o escopo dos estudos e serem mais sugestivos do ponto de vista prático da gestão para sustentabilidade.

\section{DISCUSSÕES E CONSIDERAÇÕES FINAIS}

A sustentabilidade no campo do turismo tem sido um tema relevante para gestão dos destinos. Várias perspectivas de análises vêm sendo trabalhadas e aquelas relacionadas ao consumo ainda tem recebido pouca atenção por parte dos pesquisadores, algo que se destaca aqui como problemático. $\mathrm{O}$ conceito de consumo utilizado na área de turismo em estudos sobre sustentabilidade, tende a ser amplo e muitas vezes superficial; ou ainda, tende a não existir, o que precisa ser mais bem observado. Portanto, acredita-se que seja necessário especificar o tipo de comportamento de consumo sempre relacionando às atitudes, valores, interesses, hábitos, percepções, preferências, costumes e outros aspectos individuais. O conhecimento desses comportamentos são fundamentais para o desenvolvimento de práticas concretas de preservação e manutenção de destinações turísticas, a partir da motivação e envolvimento dos visitantes.

Apesar de os estudos normalmente darem ênfase à dimensão econômica da sustentabilidade e a perspectiva de sustentabilidade de modo geral, parte significativa daqueles aqui analisados não especificam nem o contexto no qual o estudo se encontra, nem a maneira em que as dimensões estão sendo analisadas, o que dificulta o entendimento da lógica dos estudos publicados. Além disso, constatou-se que nem sempre o tipo de consumo trabalhado é especificado. Por outro lado, a tipologia de consumo sustentável tem sido a mais comum entre os estudos, o que é algo relevante para área. Apesar deste entendimento, as definições operacionais na área ainda são superficiais. Ainda assim, parece que as pesquisas brasileiras sobre consumo, turismo e sustentabilidade não trazem contribuições concretas para serem úteis à prática do turismo sustentável.

É válido destacar que mais da metade dos artigos analisados são considerados exploratórios e, aproximadamente, um quarto não apresentam especificação, características que também merece destaque. Primeiro, porque dificilmente é possível classificar um estudo como exploratório, já que normalmente os pesquisadores associam a exploração ao campo de análise e não ao aspecto teórico-metodológico. Segundo, porque todos os estudos analisados apresentam características apenas descritivas e, com isso, entende-se que essa associação indevida indica uma possível falha de interpretação metodológica.

É possível constatar que as investigações analisadas concentram-se em contextos mais amplos como turismo e desenvolvimento sustentável, impacto do turismo nas localidades, 
práticas de sustentabilidade e práticas de gestão. Apesar de se observar análises na perspectiva prática, ainda há a necessidade de se considerar os turistas como agentes do processo de preservação, isso porque o consumo associado a sustentabilidade do turismo está voltado para análise das escolhas que eles fazem ao decidir por um bem ou serviço no destino. Neste sentido, ressalta-se a ideia de se associar o ato de consumir às práticas de sustentabilidade nas destinações, sendo essa a sugestão para agenda da área.

Certamente há limitações em termos de análises e possíveis classificações iméritas por conta das características dos artigos, pois muitos deles não são claros em termos de definições e escolhas metodológicas. No entanto, o levantamento da base brasileira em um período recente mostra, de forma objetiva, um panorama de como estão as pesquisas no Brasil. Sugere-se que análises em bases internacionais sejam realizadas de forma comparativa para verificar se as pesquisas brasileiras convergem ou divergem das internacionais. Além disso, o tema pode ser mais discutido em grupos de pesquisas para que os projetos a serem desenvolvidos contemplem mais a perspectiva do consumo e prática para sustentabilidade.

\section{REFERÊNCIAS}

Bardin, L. (2009). Análise de Conteúdo. (5a ed.). Lisboa: Ed 70.

Beckerman, W. (1992). Economic growth and the environment: whose growth? Whose environment? World Development, 20(4), 481-496.

Castro, A. A. (2010). Revisão Sistemática e Meta-análise. São Paulo: MBE/UNIFESP.

Clarke M., \& Oxman, A. D. (2001). Introduction. Cochrane Reviewers' Handbook 4.1. In:

Review Manager. Version 4.1. Oxford. England: The Cochrane Collaboration.

Ciegis, R., Ciegis, R., \& Jasinskas, E. (2005). Concepts of strong comparability and commensurability versus concepts of strong and weak sustainability. Inzinerine EkonomikaEngineering economics, 45(5), 31-35.

Eden, S. E. (1993). Individual environmental responsibility and its role in public environmentalism. Environment and Planning, 25, 1743-1758.

Gil, A. C. (2008). Métodos e técnicas de pesquisa social. (6a ed.). São Paulo: Atlas.

Halkier, B. (1999). Consequences of the politicization of consumption: the example of environmentally friendly consumption practices. Journal of Environmental Policy and Planning, 1, 25-41.

Hunter, C. J. (2002). Sustainable tourism and the touristic ecological footprint. Environment, Development and Sustainability, 4(1), 7-20. 
Junqueira, L. P., Pinheiro, F. P., \& Maior, J. C. S. (2012). Sustentabilidade: a produção científica brasileira entre os anos de 2000 e 2009. Revista Científica Hermes, 6, 43-65.

Luca, M. M. M., Cardoso, V. I. C., Vasconcelos, A. C., \& Pontes, A. B. (2014). Análise da produção científica referente à temática de sustentabilidade em pesquisas da administração. RAEP Administração: Ensino \& Pesquisa, 15(3), 469-500.

Machado Jr., C., Souza, M. T. S., Ribeiro, H.C. M., Furlaneto, C. J., Silva, W. N., \& Campanario, P. M. (2013). Estudo bibliométrico da sustentabilidade ambiental: os 15 anos do Simpósio de Administração da Produção, Logística e Operações Internacionais - SIMPOI. J Health Sci Inst., 31(2), 123-131.

Manaktola, K., \& Jauhari, V. (2007). Exploring consumer attitude and behavior towards green practices in the lodging industry in India. International Journal of Contemporary Hospitality Management, 19(5), 364-377.

Melo, F. V. S., \& Farias, S. A. (2014). Sustainability as an Identity Factor of Tourist Destinations at Websites: Does the Consumer Care? Brazilian Business Review, 11(2), 135158.

Melo, F. V. S., \& Farias, S. A. (2018). Sustainability communication and its effect in consumer intention to visit a tourist destination. Tourism \& Management Studies, 14, 36-44.

Portilho, F. (2005). Consumo sustentável: limites e possibilidades de ambientalização e politização das práticas de consumo. Cadernos EBAPE.BR, 3(3), 1-12.

Silva, M. E. (2012). Consumo Sustentável: A Articulação de um constructo sob a perspectiva do desenvolvimento sustentável. Revista Eletrônica de Ciência Administrativa, 11(2), 217232.

Silva, M. E., Alves, A. P. F., \& Volkmer, G. (2014). Está chovendo no molhado? A visão acadêmica sobre o desenvolvimento sustentável em um contexto de crise. Revista de Administração da UFSM, 7, Edição Especial, 70-87.

Silva, M. E., Oliveira, A. P. M., \& Gómez, C. R. P. (2013). Indicadores de consumo consciente: uma avaliação do recifense sob a ótica do consumo sustentável. Revista Eletrônica de Ciência Administrativa, 12(2), 173-190.

Vilela, R. B., Ribeiro, A., \& Batista, N. A. (2020). Word cloud as a tool for content analysis: an application to the challenges of the professional master's degree courses in health education. Millenium, 2(11), 29-36.

\section{FORMATO PARA CITAÇÃO DESTE ARTIGO}

MELO, S. R. S., SILVA, M. E., \& MELO, F. V. S. (2021). Consumo e Sustentabilidade em Turismo: panorama, conceitos e métodos aplicados no contexto de pesquisas no Brasil. Revista de Turismo Contemporâneo, 9(2), 215-229. https://doi.org/10.21680/2357$\underline{8211.2021 v 9 n 2 I D 21641}$ 